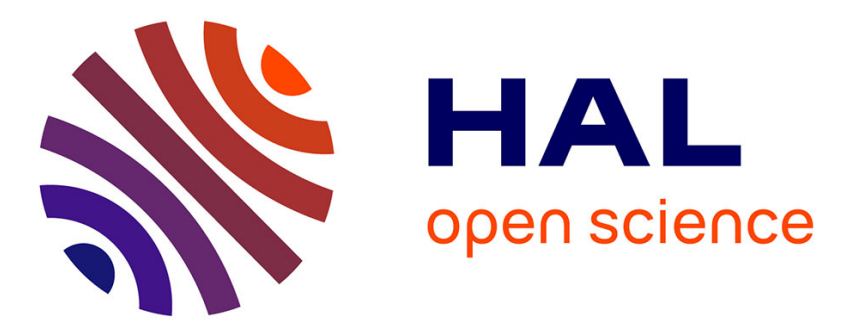

\title{
On Output Regulation in Systems with Differential Variational Inequalities (Long Version)
}

\author{
Aneel Tanwani, Bernard Brogliato, Christophe Prieur
}

\section{To cite this version:}

Aneel Tanwani, Bernard Brogliato, Christophe Prieur. On Output Regulation in Systems with Differential Variational Inequalities (Long Version). CDC 2014 - 53rd IEEE Conference on Decision and Control, Dec 2014, Los Angeles, United States. pp.TuB01.3, 10.1109/CDC.2014.7039863 . hal01065090

\section{HAL Id: hal-01065090 https://hal.science/hal-01065090}

Submitted on 17 Sep 2014

HAL is a multi-disciplinary open access archive for the deposit and dissemination of scientific research documents, whether they are published or not. The documents may come from teaching and research institutions in France or abroad, or from public or private research centers.
L'archive ouverte pluridisciplinaire HAL, est destinée au dépôt et à la diffusion de documents scientifiques de niveau recherche, publiés ou non, émanant des établissements d'enseignement et de recherche français ou étrangers, des laboratoires publics ou privés. 


\title{
On Output Regulation in Systems with Differential Variational Inequalities
}

\author{
Aneel Tanwani, Bernard Brogliato, Christophe Prieur
}

\begin{abstract}
We consider the problem of designing state feedback control laws for output regulation in a class of dynamical systems which are described by variational inequalities and ordinary differential equations. In our setup, these variational inequalities are used to model state trajectories constrained to evolve within time-varying, closed, and convex sets, and systems with complementarity relations. We first derive conditions to study the existence and uniqueness of solutions in such systems. The derivation of control laws for output regulation is based on the use of internal model principle, and two cases are treated: first, a static feedback control law is derived when full state feedback is available; In the second case, only the error to be regulated is assumed to be available for measurement and a dynamic compensator is designed. As applications, we demonstrate how control input resulting from the solution of a variational inequality results in regulating the output of the system while maintaining polyhedral state constraints. Another application is seen in designing switching signals for regulation in power converters.
\end{abstract}

\section{INTRODUCTION}

Differential variational inequalities (DVIs) provide a mathematical framework to model evolution of state trajectories which, in addition to ordinary differential equations, satisfy some algebraic relations as well. Roughly speaking, DVIs comprise an ordinary differential equation to describe the motion of the state variable, and a variational inequality (VI) that expresses the constraints, and relations that must be satisfied by the state variable. VIs are most commonly encountered in optimization, and DVIs have found applications in modeling of electrical circuits with nonsmooth devices, and mechanical systems with impacts.

While most of the work on DVIs in the recent past has focused on studying the solution theory of such systems [5], [7], [8], [21], some classical control-theoretic problems such as stability [9], [16], optimal control [4], observer and controller design [6], [17], [23] have been investigated in related system classes. The problem of output regulation for such systems, which relates to designing control laws for asymptotically tracking a reference trajectory or rejecting disturbances while respecting the relations imposed by system dynamics, was introduced in our recent work [25] and in this paper, we extend our results to a broader class of systems.

In the standard formulation of VIs [13], we consider a set-valued map, say $F$, and are interested in solving the

A. Tanwani and C. Prieur are with Gipsa-Lab (CNRS), 11 Rue des Mathématiques, BP 46, 38402 St. Martin d'Hères, France. Email: \{aneel.tanwani, christophe.prieur\}@gipsa-lab.fr. B. Brogliato is with INRIA - Rhône-Alpes, 655 Avenue de l'Europe, 38334 Montbonnot, France. Email: \{bernard.brogliato\} @inria.fr. This work has been partially supported by the LabEx PERSYVAL-Lab (ANR-11-LABX-0025-01) following inequality:

$$
\left\langle F(v), v^{\prime}-v\right\rangle \geq 0, \quad \forall v^{\prime} \in \mathcal{S},
$$

for some closed, convex, and stationary set $\mathcal{S}$. In the dynamical systems considered in this paper, we couple such VIs with ordinary differential equations, and moreover also let the set $\mathcal{S}$ be time-varying. ${ }^{1}$ Since the solutions of VIs could be abstractly represented using a set-valued operator, different tools from the theory of differential inclusions could be used to study the solutions of DVIs. We refer the reader to a recent paper [21] for results and literature in this direction. In this paper, we also propose results on existence and uniqueness of solutions for a particular system class, which is based on the theory of evolution equations with maximal monotone operators [3]. While VIs essentially describe a maximal monotone operator, in many practical systems, these operators only appear after certain linear transformations, and it is not evident that such transformations preserve maximality, or even monotonicity. Our approach extends the work of [5] by relaxing certain assumptions (that describe the transformation) required to show the existence of solutions. In a recent paper [10], it is shown that if the transformation matrices satisfy certain LMI (related to the passivity assumption in control theory), then maximal monotonicity is preserved. We generalize the results of [10] by considering nonlinear globally Lipschitz vector fields in system description, and consider nonstationary sets $\mathcal{S}$. The proof worked out in this case is also completely different than the work of [10].

The second main contribution of this paper comes in applying these results to study the problem of output regulation. Our approach is based on the pioneering work of [14] which proposed simple algebraic criteria for output regulation in multivariable linear time-invariant (LTI) systems. In [14], it is assumed that the plant under consideration is driven by the output of an LTI exosystem that models the dynamics of the reference trajectories and/or disturbances. Intuitively speaking, the proposed control input that achieves the output regulation comprises a feedback component to make the closed-loop dynamics stable and an additional open-loop component that shapes the steady state of the plant. The derivation of the open-loop component of the control input requires the exact knowledge of the exosystem dynamics, and hence the approach is termed as internal model principle. A large amount of work has followed on the problem of output regulation using internal model principle in more general

\footnotetext{
${ }^{1}$ In the paper [21], the case of time-varying $\mathcal{S}$ is termed as quasi-DVI, but in this paper we do not make this distinction as we only consider the time-varying case and our results obviously hold when $\mathcal{S}$ is stationary.
} 
contexts and more recently in the context of discontinuous and hybrid systems, see [11], [12], [15], [19] and references therein. This paper could be seen as extending this principle for a class of nonsmooth systems expressed as DVIs.

In our results on output regulation, we will restrict ourselves to linear systems. The sets considered in the description of VIs for the exosystem and the plant are assumed to be the same but the mappings used to describe the relations could be different. We derive sufficient conditions under which there exists a control input that achieves output regulation while maintaining state constraints. In addition to the classical regulator synthesis equations, additional conditions are needed in our work to generate a dissipative relation between the multivalued part and the output regulation error. These additional conditions also guarantee that the closedloop system is well-posed, that is, it admits a unique solution which is an important consideration for designing controllers for such class of systems. We study two cases for control synthesis depending on how much information is available to the controller. In the first case, it is assumed that the entire states of the plant and the exosystem are available and thus, a static controller is designed to achieve output regulation. In the second case, it is assumed that only the regulation error (which needs to converge to zero) is available and in that case a dynamic compensator is designed.

The rest of the paper is organized as follows: In Section II, we define the system class, formulate the problem of output regulation that we consider, and introduce some basic results from convex analysis. These results are used to develop a result on existence and uniqueness of solutions for proposed system class in Section III. The design of static state feedback is considered in Section IV, followed by the design of a dynamic compensator in Section V. The proofs of some intermediate results are collected in the Appendix.

\section{PRELIMINARIES}

\section{A. Differential Variational Inequalities}

Consider a set-valued mapping $\mathcal{S}:[0, \infty) \rightrightarrows \mathbb{R}^{d_{s}}$, and assume that $\mathcal{S}(t)$ is closed, convex, and nonempty, for each $t \geq 0$. The class of differential variational inequalities considered in this paper are described as follows:

$$
\begin{aligned}
& \dot{x}(t)=f(t, x)+G \lambda(t) \\
& v(t)=H x(t)+J \lambda(t), \quad v(t) \in \mathcal{S}(t), \\
& \left\langle v^{\prime}-v(t), \lambda(t)\right\rangle \geq 0, \quad \forall v^{\prime} \in \mathcal{S}(t) .
\end{aligned}
$$

In the above equation $x(t) \in \mathbb{R}^{n}$ denotes the state, $\lambda(t), v(t) \in \mathbb{R}^{d_{s}}$, the vector field $f:[0, \infty) \times \mathbb{R}^{n} \rightarrow$ $\mathbb{R}^{n}$ is absolutely continuous in first argument, and globally (and uniformly with respect to time) Lipschitz in second argument, $G \in \mathbb{R}^{n \times d_{s}}, H \in \mathbb{R}^{d_{s} \times n}, J \in \mathbb{R}^{d_{s} \times d_{s}}$ are constant matrices, and $J$ is positive semidefinite.

In the standard references on variational inequalities, the multivalued mapping $\mathcal{S}(\cdot)$ is assumed to be stationary [13], [21]. To make connections with the standard formulation of evolution equations with time-varying domains [2], [20], it is seen that (1b), (1c) could be compactly written as:

$$
\lambda(t) \in-\mathcal{N}_{\mathcal{S}(t)}(H x(t)+J \lambda(t)),
$$

where $\mathcal{N}_{\mathcal{S}}(t)(v(t))$ denotes the normal cone to a convex set at $v(t) \in \mathcal{S}(t)$, and is defined as:

$$
\mathcal{N}_{\mathcal{S}(t)}(v(t)):=\left\{\lambda \in \mathbb{R}^{d_{s}} \mid\left\langle\lambda, v^{\prime}-v(t)\right\rangle \geq 0, \forall v^{\prime} \in \mathcal{S}(t)\right\},
$$

and as convention we let $\mathcal{N}_{\mathcal{S}}(t)(v(t)):=\emptyset$, for $v(t) \notin \mathcal{S}(t)$.

In what follows, we will use the standard notations, int, and rint to denote the interior, and the relative interior of a set respectively. The domain, range, and kernel of an operator are denoted by dom, rge, and ker respectively.

\section{B. Problem Formulation}

As stated in the introduction, we basically consider two problems related to system class (1).

1) Well-posedness of DVI (1): First, we are interested in knowing under what conditions on the system dynamics, a unique solution exists in the following sense:

Definition 1: For each initial condition $x(0)$ satisfying $H x(0) \in \mathcal{S}(0)+\operatorname{rge} J$, there exists a locally absolutely continuous function $x:[0, \infty) \rightarrow \mathbb{R}^{n}$, such that $x(\cdot)$ satisfies (1a) for (Lebesgue-almost) every $t \geq 0$, and $H x(t) \in$ $\mathcal{S}(t)+\operatorname{rge} J$.

2) Output Regulation: For this problem, we restrict ourselves to the case of linear vector fields, and the system class in particular is defined as follows:

$$
\begin{gathered}
\dot{x}(t)=A x(t)+B u(t)+F x_{r}(t)+G \lambda(t) \\
\lambda(t) \in-\mathcal{N}_{\mathcal{S}(t)}(H x(t)+J \lambda(t))
\end{gathered}
$$

where $x_{r}:[0, \infty) \rightarrow \mathbb{R}^{d_{r}}$ is the reference signal that is generated from the following equations:

$$
\begin{gathered}
\dot{x}_{r}(t)=A_{r} x_{r}(t)+G_{r} \lambda_{r}(t) \\
\lambda_{r}(t) \in-\mathcal{N}_{\mathcal{S}(t)}\left(H_{r} x_{r}(t)+J_{r} \lambda_{r}(t)\right) .
\end{gathered}
$$

The output regulation variable $w(\cdot)$ is defined as:

$$
w(t)=C x(t)+D x_{r}(t) .
$$

It will be assumed throughout the paper that system (4) admits a solution (not necessarily unique) in the sense of Definition 1. We say that the output regulation is achieved if there exists a control input $u(\cdot)$ such that the following properties are satisfied:

- Well-posedness: For each initial condition $x(0)$ satisfying $H x(0) \in \mathcal{S}(0)+\operatorname{rge} J$, there exists a unique solution in the sense of Definition 1.

- Regulation: It holds that $\lim _{t \rightarrow \infty} w(t)=0$.

- Closed-loop stability: The plant and controller dynamics have a globally asymptotically stable equilibrium at the origin when $x_{r} \equiv 0$.

\section{Motivation}

The solution theory for system (1) could be useful in many aspects since several electrical and mechanical systems are modeled using this framework. To motivate the output regulation, we mention two possible applications of the proposed problem. 
1) Viability Control and Regulation: As first application, we consider the problem of finding a control input which maintains predefined constraints on the state trajectories of a dynamical system while achieving output regulation. Stated more precisely, suppose that we are given a plant described as:

$$
\dot{x}(t)=A x(t)+B u(t)+F x_{r}
$$

and we would like to find a control $u(\cdot)$ which not only tracks a reference trajectory generated by the exosystem of form (4), but also results in the state satisfying the constraint that $H x(t) \in \mathcal{S}(t)$, for all $t \geq 0$, where $\mathcal{S}(\cdot)$ is some predefined closed and convex set-valued map. This could be achieved by decomposing $u$ as $u:=u_{\text {reg }}+u_{\lambda}$, where we choose $u_{\lambda}(t)$ as the solution of the following variational inequality:

$$
u_{\lambda}(t)^{\top}\left(v^{\prime}-H x(t)\right) \geq 0, \quad \forall v^{\prime} \in \mathcal{S}(t) .
$$

This choice of control input transforms the plant equation (6) as follows:

$$
\dot{x}(t)=A x(t)+B u_{\mathrm{reg}}(t)+B u_{\lambda}(t)+F x_{r}(t) .
$$

For any $u_{\lambda}(\cdot)$ that satisfies (7), it now holds for the trajectories of the closed-loop system (8) that $v(t):=H x(t) \in \mathcal{S}(t)$, provided that the system (8) is well-posed. The case of $\mathcal{S}(\cdot)$ being a time-varying polytope was also considered as a special case in our previous work [25]. In that case, (7) is formulated as a linear complementarity problem which could be solved very efficiently using standard softwares.

2) Regulation in Power Converters: A large number of electrical circuits with nonsmooth devices (diodes, switches, etc.), such as power converters, are modeled using complementarity relations which is a special kind of variational inequality when the set-valued map $\mathcal{S}(t)=\mathcal{K}$, where $\mathcal{K} \subseteq$ $\mathbb{R}^{d_{K}}$ is some closed convex cone (see [1], [26] for examples). Let $\mathcal{K}^{*}$ denote the dual cone to $\mathcal{K}$, defined as:

$$
\mathcal{K}^{*}:=\left\{\bar{v} \in \mathbb{R}^{d_{K}} \mid\langle\bar{v}, w\rangle \geq 0, \forall w \in \mathcal{K}\right\} .
$$

Our framework allows us to consider the models of electrical systems of the following form:

$$
\begin{gathered}
\dot{x}(t)=A x(t)+B_{1} u_{1}(t)+F_{1} x_{r}(t)+G \lambda(t) \\
v(t)=H x(t)+J \lambda(t)+F_{2} x_{r}(t)+B_{2} u_{2}(t) \\
\mathcal{K} \ni v(t) \perp \lambda(t) \in \mathcal{K}^{*},
\end{gathered}
$$

where $u_{1}(\cdot)$ and $u_{2}(\cdot)$ are control inputs that need to be designed to solve the regulation problem. Since $\mathcal{K}$ is assumed to be a cone, the cone complementarity problem (9c) is equivalent to [13, Proposition 1.1.3]:

$$
\lambda(t) \in-\mathcal{N}_{\mathcal{K}}(v(t)) .
$$

If we let $\overline{\mathcal{K}}(t):=\mathcal{K}-F_{2} x_{r}(t)-B_{2} u_{2}(t)$, so that $\bar{v}(t) \in \overline{\mathcal{K}}(t)$ if and only if $\bar{v}(t)+F_{2} x_{r}(t)+B_{2} u_{2}(t) \in \mathcal{K}$, then (9b), (9c) are equivalently written as:

$$
\begin{gathered}
\bar{v}(t)=H x(t)+J \lambda(t) \\
\lambda(t) \in-\mathcal{N}_{\overline{\mathcal{K}}(t)}(\bar{v}(t))
\end{gathered}
$$

and hence the system (9) is of the same form as (3).

\section{Basic Results: Convex Analysis}

In this section, we recall some basic results from convex analysis which are used in the subsequent sections for deriving main results of this paper. One can consult standard references, such as [22], for the results given here.

A set-valued map $\Phi(\cdot)$ is called maximal monotone if for each $x_{1}, x_{2} \in \operatorname{dom}(\Phi)$, and $y_{i} \in \Phi\left(x_{i}\right), i=1,2$, we have $\left\langle y_{2}-y_{1}, x_{2}-x_{1}\right\rangle \geq 0$, and the graph of $\Phi$ cannot be extended any further while satisfying the monotone property. We will not use the definition in its entirety. The monotone property is important and will be used repeatedly in analysis. Another important thing to know is that there is a vast literature on the solution theory of differential inclusions where the multivalued operator on the right-hand side is maximal monotone [3].

The following result allows us to draw connection between system (1a), (2) and the theory of maximal monotone operators.

Proposition 1: Consider a nonempty, closed and convex set $\mathcal{S}$ and let $\psi_{\mathcal{S}}(\cdot)$ denote its indicator function, that is, $\psi_{\mathcal{S}}(v)=0$, if $v \in \mathcal{S}$ and $\psi_{S}(v)=+\infty$ otherwise; Then

1) it holds that $\partial \psi_{\mathcal{S}}(v)=\mathcal{N}_{\mathcal{S}}(v)$,

2) and $\mathcal{N}_{\mathcal{S}}(\cdot)$ is a maximal monotone operator.

In our approach, we would like to express (1) as a differential inclusion by replacing $\lambda$ with a set-valued operator. In order to do that, one can see from (2) that we would need to define the "inverse" of the normal cone operator. The theory of conjugate functions (or Legendre-Fenchel transforms) [22, Chapter 11] allows us to make this connection.

Definition 2: For a function $g: \mathbb{R}^{n} \rightarrow[-\infty,+\infty]$, the function $g^{*}: \mathbb{R}^{n} \rightarrow[-\infty,+\infty]$ defined as:

$$
g^{*}(\eta):=\sup _{v}\{\langle\eta, v\rangle-g(v)\}
$$

is called the conjugate of $g$. For a closed convex set $\mathcal{S}$, the conjugate of the indicator function $\psi_{\mathcal{S}}(\cdot)$ is the support function $\sigma_{\mathcal{S}}(\cdot)$ defined as:

$$
\sigma_{\mathcal{S}}(\eta)=\max _{v \in \mathcal{S}}\langle v, \eta\rangle
$$

We now recall the following fundamental result:

Proposition 2 ([22, Proposition 11.3]): For any proper, lower semicontinuous, convex function $g(\cdot)$, one has $\partial g^{*}=$ $(\partial g)^{-1}$ and $\partial g=\left(\partial g^{*}\right)^{-1}$. That is,

$$
\eta \in \partial g(v) \Longleftrightarrow v \in \partial g^{*}(\eta)
$$

In particular, for a closed and convex set $\mathcal{S}$ :

$$
\eta \in \mathcal{N}_{\mathcal{S}}(v) \Longleftrightarrow v \in \partial \sigma_{\mathcal{S}}(\eta) .
$$

\section{INCLUSION WITH MAXIMAL MONOTONE OPERATOR}

There is a considerable amount of literature on the solution theory for differential inclusions, depending on the structure of the set-valued map on the right-hand side. One particular class of set-valued maps, which are interesting from the point of analysis and applications, are maximal monotone operators, and the solution theory for differential inclusions with such operators has been well-studied since the work of Brézis [3]. For our setup, such inclusions are of particular 
interest because we will now show that, when the set $\mathcal{S}(t)$ is closed and convex valued, for each $t \geq 0$, then equation (1) can be equivalently written as a differential inclusion with time-varying maximal monotone operator plus a globally Lipschitz vector field on the right-hand side.

To see this, we use Propositions 1 and 2, and describe the relations in (1a), (1b), using a set-valued map for $\lambda(t)$ as follows:

$$
\begin{aligned}
& \left\langle v^{\prime}-v(t), \lambda(t)\right\rangle \geq 0, \quad \forall v^{\prime} \in \mathcal{S}(t) \\
\Longleftrightarrow & \lambda(t) \in-\partial \psi_{S(t)}(H x(t)+J \lambda(t)) \\
\Longleftrightarrow & H x(t)+J \lambda(t) \in \partial \sigma_{\mathcal{S}(t)}(-\lambda(t)) \\
\Longleftrightarrow & H x(t) \in\left(\partial \sigma_{\mathcal{S}(t)}+J\right)(-\lambda(t)) \\
\Longleftrightarrow & \lambda(t) \in-\left(\partial \sigma_{\mathcal{S}(t)}+J\right)^{-1}(H x(t))
\end{aligned}
$$

Letting $\Phi(t, H x(t)):=\left(\partial \sigma_{\mathcal{S}(t)}+J\right)^{-1}(H x(t))$, system (1) can be equivalently written as the following differential inclusion:

$$
\dot{x}(t) \in f(t, x)-G \Phi(t, H x(t)) .
$$

It is an easy exercise to show that the operator $\Phi(t, \cdot)$ is maximal monotone for each $t \geq 0$ (see also proof of Lemma 1 in the Appendix) but it is not true in general that $G \Phi(t, H \cdot)$ is also maximal monotone. If it is assumed that $f(t, x)=A x+u(t)$, and that the LTI system defined using the matrices $(A, G, J, H)$ is passive and $\Phi$ is timeindependent, then the maximal monotonicity of the multivalued operator on the right-hand side of (12) was proven in [10]. Our goal in this section is to generalize this result for the class of systems (12), and the contribution of what follows could be seen in following two regards:

- A direct approach (different than [10]) to transform the right-hand side of (12) into a maximal monotone operator (with a minus sign) and a Lipschitz vector field, the solutions for which can be derived using existing results.

- Generalize the system class by addressing time-dependent sets and nonlinear vector fields.

\section{A. Solution of Differential Inclusion (12)}

Compared to the standard formulations of evolution equations with maximal monotone operators, the primary difficulty in studying the well-posedness of (12) is that the operator $G \Phi(t, H \cdot)$ may not necessarily be monotone for some arbitrary matrices $G$ and $H$ because multiplication with matrices does not preserve monotonicity. Secondly, the righthand side depends on time and we need to impose some constraints on the evolution of the set-valued map $\mathcal{S}(\cdot)$ along with some regularity assumptions on $f(\cdot, x)$. The main result overcoming these difficulties is stated as follows:

Theorem 1: Assume that the following holds:

(A1) The matrix $J$ is positive semidefinite and there exists a symmetric positive definite matrix $P$ such that $\operatorname{ker}(J+$ $\left.J^{\top}\right) \subseteq \operatorname{ker}\left(P G-H^{\top}\right)$.

(A2) For each $t \geq 0$, the range space of $H$, denoted as rge $H$, cannot be separated from $\mathcal{S}(t)+\operatorname{rge} J$.

(A3) The mapping $\mathcal{S}:[0, \infty) \rightrightarrows \mathbb{R}^{d_{s}}$ is closed and convex valued with nonempty relative interior for each $t \geq 0$, and varies in an absolutely continuous manner with time, that is, there exists an absolutely continuous function $\nu(\cdot):[0, \infty) \rightarrow \mathbb{R}_{+}$, such that,

$$
\left|d\left(v, \mathcal{S}\left(t_{1}\right)\right)-d\left(v, \mathcal{S}\left(t_{2}\right)\right)\right| \leq\left|\nu\left(t_{1}\right)-\nu\left(t_{2}\right)\right|, \forall t_{1}, t_{2} \geq 0 .
$$

(A4) The function $f(t, \cdot)$ is globally Lipschitz (uniformly in time), that is, there exists a constant $\mu>0$ such that for each $t \geq 0$ :

$$
\left|f\left(t, x_{1}\right)-f\left(t, x_{2}\right)\right| \leq \mu\left|x_{1}-x_{2}\right|, \quad \forall x_{1}, x_{2} \in \mathbb{R}^{n},
$$

and $f(\cdot, x)$ is absolutely continuous for each $x \in \mathbb{R}^{n}$.

Then there exists a unique solution to (12), and hence (1), in the sense of Definition 1.

Discussions:

1) If $J=0$, then (A1) basically implies that $P J=H^{\top}$, which was the assumption used to deduce the maximal monotonicity of the multivalued operator in [5]. Also, if the quadruple $(A, B, J, H)$ is passive, then the assumption (A1) automatically holds and this fact was also used in [10].

2) The constraint qualification (A2) is somewhat a standard assumption when dealing with problems on variational inequalities, and is introduced to preserve maximality under the composition function.

3) The bound on the variation of $\mathcal{S}(\cdot)$ is introduced to obtain absolutely continuous trajectories. It basically imposes the constraint that the Hausdorff distance between the sets $\mathcal{S}\left(t_{1}\right)$ and $\mathcal{S}\left(t_{2}\right)$ is relatively small for $\left|t_{1}-t_{2}\right|$ sufficiently small. One could also relax the function $\nu(\cdot)$ to be continuous, or a function with locally bounded variation, in which case the resulting state trajectory will also be in the class of functions with locally bounded variation. However, this will only complicate the development as one has to interpret (12) as a measure differential inclusion. Nonetheless, we conjecture that most of the development carried out in the sequel will also carry over to that case.

4) The Lipschitz condition on the function $f(t, \cdot)$ is imposed because the inclusions with maximal monotone operators and Lipschitz perturbations are well-posed [3].

Proof of Theorem 1: The proof of Theorem 1 is based on several intermediate results which we will state as lemmas. The proofs of these lemmas are given in the Appendix. $^{2}$

Lemma 1: For each $t \geq 0$, the operator $\Phi(t, \cdot)=$ $\left(\partial \sigma_{\mathcal{S}(t)}+J\right)^{-1}(\cdot)$ is maximal monotone and $\operatorname{dom} \Phi(t, \cdot)=$ $\mathcal{S}(t)+\operatorname{rge} J$.

Lemma 2: Consider $\eta_{1}, \eta_{2} \in \Phi(t, H x)$, then $\eta_{1}-\eta_{2} \in$ $\operatorname{ker}\left(J+J^{\top}\right)$.

For each $\eta_{\alpha} \in \Phi(t, H x)$ (with $t$ and $x$ fixed), let $\eta^{\text {im }}$ denote the projection of $\eta_{\alpha}$ on the orthogonal complement of $\operatorname{ker}\left(J+J^{\top}\right)$, denoted as $\eta^{\mathrm{im}}=\mathcal{P}_{J}\left(\eta_{\alpha}\right)$, then using Lemma 2, $\eta^{\mathrm{im}}$ is uniquely defined and we can write

$$
\eta_{\alpha}:=\eta^{\mathrm{im}}+\eta_{\alpha}^{\mathrm{ker}}
$$

\footnotetext{
${ }^{2}$ In the proof of Theorem 1 and the Appendix, we will use the notation $\eta(t)$ to denote an element of the set $\Phi(t, H x)$. From (11e), it is seen that the variable $\eta$ and $\lambda$ only differ by a sign change.
} 
for some $\eta_{\alpha}^{\mathrm{ker}} \in \operatorname{ker}\left(J+J^{\top}\right)$. Since we have assumed that $\operatorname{ker}\left(J+J^{\top}\right) \subseteq \operatorname{ker}\left(P G-H^{\top}\right)$, we must have $G \eta_{\alpha}^{k e r}=$ $P^{-1} H^{\top} \eta_{\alpha}^{\text {ker }}$. This allows us to rewrite (12) as follows:

$$
\begin{aligned}
\dot{x}(t) & =f(t, x)-G \eta^{\mathrm{im}}(t)-P^{-1} H^{\top} \eta_{\alpha}^{\mathrm{ker}}(t) \\
& =f(t, x)+\left(P^{-1} H^{\top}-G\right) \mathcal{P}_{J}\left(\eta_{\alpha}(t)\right)-P^{-1} H^{\top} \eta_{\alpha}(t) \\
\eta_{\alpha}(t) & \in \Phi(t, H x(t)) .
\end{aligned}
$$

Let $R$ denote the square root of the matrix $P$ in (A1), so that $R$ is also positive definite and symmetric. Introduce the coordinate transformation $z=R x$, then in the new coordinates, (13) is written as:

$$
\begin{aligned}
& \dot{z}(t)= R f\left(t, R^{-1} z\right)+\left(R^{-1} H^{\top}-R G\right) \mathcal{P}_{J}\left(\eta_{\alpha}(t)\right) \\
&-R^{-1} H^{\top} \eta_{\alpha}(t) \\
& \eta_{\alpha}(t) \in \Phi\left(t, H R^{-1} z(t)\right) .
\end{aligned}
$$

We will next use the following two results:

Lemma 3: The operator $\mathcal{P}_{J}(\Phi(t, H \cdot))$ is single-valued and Lipschitz continuous.

Lemma 4 ([22, Theorem 12.43]): The multivalued operator $R^{-1} H^{\top} \Phi\left(t, H R^{-1} \cdot\right)$ is maximal monotone, for each $t \geq 0$, if $\operatorname{rge}\left(H R^{-1}\right) \cap \operatorname{rint}(\operatorname{dom}(\Phi(t, \cdot))) \neq \emptyset$, which holds in particular under the constraint qualification (A2).

As a result of these two lemmas, one can now write (14) as:

$$
\dot{z}(t) \in g(t, z)-\Psi(t, z)
$$

where $g(t, z)=R f\left(t, R^{-1} z\right)-\left(R^{-1} H^{\top}-R G\right) \mathcal{P}_{J}\left(\eta_{\alpha}(t)\right)$ is globally Lipschitz (in second argument) and $\Psi(t, z)=$ $R^{-1} H^{\top} \Phi\left(t, H R^{-1} z(t)\right)$ is maximal monotone for each $t \geq$ 0 . Finally, let

$$
\bar{\Psi}(t, z):=\Psi(t, z)+g(t, z)-\bar{\mu} x,
$$

where $\bar{\mu}>0$ denotes the Lipschitz constant of $g(t, \cdot)$. It is easy to check that $\bar{\Psi}(t, z)$ is also maximal monotone. Inclusion (15) is now equivalent to:

$$
\dot{z}(t) \in \bar{\mu} x-\bar{\Psi}(t, z) .
$$

One can now deduce the solution to (16) in the sense of Definition 1 by combining the results from [3, Chapter 3] and [18]. The precise arguments are worked out in the proof of following lemma given in the Appendix.

Lemma 5: For each $t \geq 0$, and there exists a unique locally absolutely continuous solution $z(\cdot)$, that satisfies (16) for almost every $t \geq 0$.

Since we have only introduced bijective operations in arriving from system (1) to (16), the conclusion also holds for system (1).

\section{Full State Feedback and Static Control}

We will now use the results on the well-posedness of system (1) to solve the output regulation problem. In this section, it is assumed that all the states of the plant (1) and (4) are available for feedback and thus a control input with static state feedback can be designed which achieves the stability and regulation. In the formulation of our results, the following terminology is used: A quadruple of matrices
$(A, B, C, D)$ is called strictly passive if there exist a scalar $\gamma>0$ and a symmetric positive definite matrix $P$ such that

$$
\left[\begin{array}{cc}
A^{\top} P+P A+\gamma P & P B-C^{\top} \\
B^{\top} P-C & -\left(D+D^{\top}\right)
\end{array}\right] \leq 0 .
$$

Theorem 2: Consider systems (3), (4) under assumptions (A2) and (A3). Suppose that a matrix $K$ renders the triplet $(A+B K, G, H, J)$ strictly passive, and that there exist matrices $\Pi \in \mathbb{R}^{n \times d_{r}}$ and $M \in \mathbb{R}^{d_{u} \times d_{r}}$ such that

$$
\begin{gathered}
\Pi A_{r}=A \Pi+B M+F \quad \text { and } \quad C \Pi+D=0 \\
\Pi G_{r}=G, \quad H \Pi=H_{r} \quad \text { and } \quad J_{r}=J .
\end{gathered}
$$

Then the output regulation problem is solvable with the following static feedback control law:

$$
u(t)=K x(t)+(M-K \Pi) x_{r}(t) .
$$

Remark 1: In the work of [14], the control law (19) was proposed to solve the output regulation problem in LTI systems, where $\Pi$ and $M$ were obtained as solution of (18a) only, and $K$ is any matrix that makes $(A+B K)$ Hurwitz. The strict passivity requirement, and additional conditions on the matrix $\Pi$ in (18b) are required in the well-posedness and stability analyses for the class of systems considered in this paper.

Proof: a) Well-posedness: With control input (19), the closed-loop system is written as

$$
\begin{gathered}
\dot{x}(t)=(A+B K) x(t)+(F+B M-B K \Pi) x_{r}(t)+G \lambda(t) \\
\lambda \in-\mathcal{N}_{\mathcal{S}(t)}(H x(t)+J \lambda(t)) .
\end{gathered}
$$

Since $(A+B K, G, H, J)$ is assumed to be strictly passive, the matrix $J$ is positive semidefinite and $\operatorname{ker}\left(P G-H^{\top}\right) \subseteq$ $\operatorname{ker}\left(J+J^{\top}\right)$. All the remaining hypothesis of Theorem 1 hold by construction, and hence the closed-loop system exhibits a unique solution.

b) Regulation: Let $\Pi$ be the matrix that satisfies (18) and introduce the variable $e=x-\Pi x_{r}$. The output regulation is achieved if we can show that $\lim _{t \rightarrow \infty} e(t)=0$, since

$$
\begin{aligned}
w(t) & =C x(t)+D x_{r}(t) \\
& =C x(t)-C \Pi x_{r}(t)=C e(t) .
\end{aligned}
$$

To show that $e(t) \rightarrow 0$ as $t \rightarrow \infty$, we observe that

$$
\begin{aligned}
& \dot{e}(t)=(A+B K) x(t)+(F+B M-B K \Pi) x_{r}(t) \\
& +G \lambda(t)-\Pi A_{r} x_{r}(t)-\Pi G_{r} \lambda_{r}(t) \\
& =(A+B K) x(t)-(\underbrace{\Pi A_{r}-F-B M}_{=A \Pi}+B K \Pi) x_{r}(t) \\
& +G \lambda(t)-\Pi G_{r} \lambda_{r}(t) \\
& =(A+B K) x(t)-(A+B K) \Pi x_{r}(t) \\
& +G \lambda(t)-\Pi G_{r} \lambda_{r}(t) \\
& =(A+B K) e(t)+G\left(\lambda(t)-\lambda_{r}(t)\right) .
\end{aligned}
$$

Now introduce the Lyapunov function $V(e)=e^{\top} P e$, so that the following holds for almost all $t \geq 0$ :

$$
\begin{aligned}
\dot{V}(e(t))= & e(t)^{\top}\left((A+B K)^{\top} P+P(A+B K)\right) e(t) \\
& +2 e(t)^{\top} P G\left(\lambda(t)-\lambda_{r}(t)\right) \\
\leq- & \gamma e(t)^{\top} P e(t) \\
& +2\left\langle\lambda(t)-\lambda_{r}(t), H e(t)+J\left(\lambda(t)-\lambda_{r}(t)\right)\right\rangle
\end{aligned}
$$


where we used the passivity of $(A+B K, G, H, J)$ to arrive at the last inequality. Recalling that $\mathrm{He}(t)=H x(t)-$ $H \Pi x_{r}(t)=H\left(x(t)-x_{r}(t)\right)$, and $J_{r}=J$, the monotonicity of the normal cone operator leads to

$$
\left\langle\lambda(t)-\lambda_{r}(t), H\left(x(t)-x_{r}(t)\right)+J\left(\lambda(t)-\lambda_{r}(t)\right)\right\rangle \leq 0 .
$$

It thus follows that $\dot{V}(e(t))<0$ for all $e(t) \neq 0$, and thus $e(t) \rightarrow 0$ as $t \rightarrow \infty$.

Closed-loop stabilization: Following the same calculations as above, it is seen that the derivative of $V(\cdot)$ along the dynamics of (3) with $u$ in (19) satisfies

$$
\dot{V}(x(t)) \leq-\gamma V(x(t))+x(t)^{\top} P(F+B(M-K \Pi)) x_{r}(t)
$$

and it follows that $x(t) \rightarrow 0$ as $t \rightarrow \infty$, when $x_{r} \equiv 0$.

Example 1: As an illustration of Theorem 2, let us consider an example in the context of application discussed in $\S$ II-C.1. The plant to be controlled is a second order LTI system described by the equations

$$
\dot{x}_{1}=-0.1 x_{1}+x_{2} ; \quad \dot{x}_{2}=u .
$$

The exosystem is defined as the following linear complementarity system:

$$
\begin{gathered}
\dot{x}_{r}:=\left(\begin{array}{c}
\dot{x}_{r 1} \\
\dot{x}_{r 2}
\end{array}\right)=\left[\begin{array}{cc}
-0.1 & 1 \\
-2 & 1
\end{array}\right] x_{r}+\left[\begin{array}{cc}
0 & 0 \\
-1 & 1
\end{array}\right] \lambda_{r} \\
0 \leq \lambda_{r} \perp\left(\begin{array}{c}
-x_{r 2} \\
x_{r 2}
\end{array}\right)+\left(\begin{array}{l}
1 \\
1
\end{array}\right) \geq 0 .
\end{gathered}
$$

Consider the set $\mathcal{S}:=\{z \in \mathbb{R}: z+1 \geq 0\}$ and the matrix $H:=\left[\begin{array}{cc}0 & -1 \\ 0 & 1\end{array}\right]$, then following the discussion in $\S$ II-C (see also $[25, \S 5]$ ), the relation (20) is equivalently expressed as $\lambda_{r} \in-\mathcal{N}_{\mathcal{S} \times \mathcal{S}}\left(H x_{r}\right)$. We are interested in designing a control input $u$, such that $\lim _{t \rightarrow \infty}\left|x_{2}(t)-x_{r 2}(t)\right|=0$, and $\forall t \geq$ $0,\left|x_{2}(t)\right| \leq 1$, or equivalently $H x(t) \in \mathcal{S} \times \mathcal{S}$. Verbally speaking, the exosystem has been chosen so that the plot of $x_{r 2}$ (versus time) resembles a sine wave clipped at the value 1, see Fig. 1 . The control objective is to guarantee $\left|x_{2}(t)\right| \leq 1$ and that $x_{2}$ converges asymptotically to $x_{r 2}$. Decomposing the input as $u:=u_{\text {reg }}+u_{\lambda}$ results in the closed-loop system of the form (8). In the notation of Theorem 2, we let $\Pi=$ $I_{2 \times 2}, P=\left[\begin{array}{ll}2 & 0 \\ 0 & 1\end{array}\right], K=\left[\begin{array}{ll}-2 & -2\end{array}\right]$, and $M=\left[\begin{array}{ll}-2 & 1\end{array}\right]$, so that $u_{\text {reg }}(t):=-2 x_{1}(t)-2 x_{2}(t)+3 x_{r 2}(t)$ follows from (19). The discontinuous component of the input ${ }^{3} u_{\lambda}:=-u_{\lambda 1}+u_{\lambda 2}$ is obtained as a solution of the following complementarity problem:

$$
0 \leq\left(\begin{array}{l}
u_{\lambda 1} \\
u_{\lambda 2}
\end{array}\right) \perp\left(\begin{array}{c}
-x_{2} \\
x_{2}
\end{array}\right)+\left(\begin{array}{l}
1 \\
1
\end{array}\right) \geq 0 .
$$

The results of the simulation are shown in Fig. 1.

\footnotetext{
${ }^{3}$ We are implicitly using the fact that the two constraints imposed in this problem, $x_{2} \leq 1$ and $x_{2} \geq-1$, are not active simultaneously. Thus, the complementarity formulation (21) ensures that $u_{\lambda}=-u_{\lambda 1}$ if $x_{2}=1$, and $u_{\lambda}=u_{\lambda 2}$ if $x_{2}=-1$, otherwise $u_{\lambda}=0$.
}

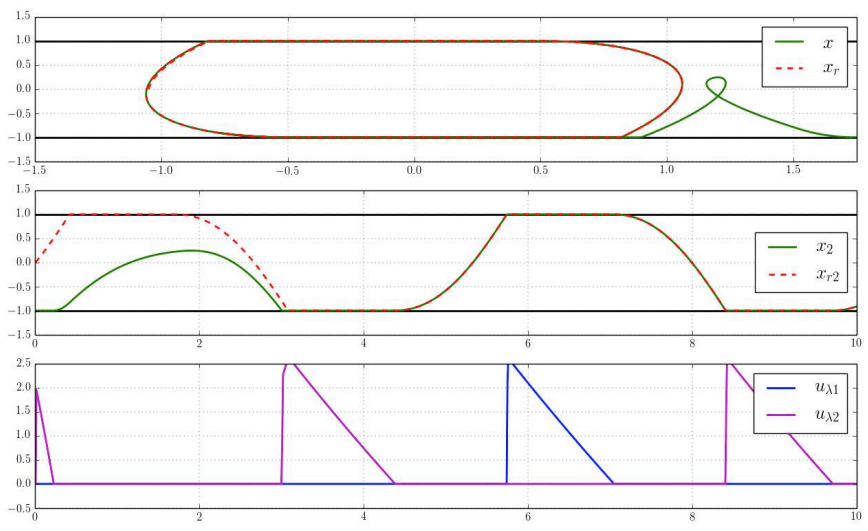

Fig. 1. The top plot shows the phase portrait of the trajectories of the plant and the exosystem. The middle plot confirms that $x_{2}$ converges to $x_{r 2}$ while staying within the set $\mathcal{S}$. The bottom plot shows the values of discontinuous component of the control input which only become nonzero when $x$ is on the boundary of the set $\mathcal{S}$.

\section{ERror FeEdback AND Dynamic COMPENSATOR}

In this section, it will no longer be assumed that the states $x(\cdot)$ and $x_{r}(\cdot)$ are available for feedback, but only the regulation error $w(\cdot)$ is available to the controller. Our approach is based on the certainty equivalence principle where we first design an estimator for the state variables $x(\cdot)$ and $x_{r}(\cdot)$ and then define the control law as a function of these estimates.

Towards this end, the estimator we propose is defined as follows:

$$
\begin{gathered}
\left(\begin{array}{c}
\dot{\hat{x}} \\
\hat{\hat{x}}_{r}
\end{array}\right)=\left[\left(\begin{array}{cc}
A & F \\
0 & A_{r}
\end{array}\right)-\left(\begin{array}{c}
L_{0} \\
L_{1}
\end{array}\right)\left(\begin{array}{ll}
C & D
\end{array}\right)\right]\left(\begin{array}{c}
\hat{x} \\
\hat{x}_{r}
\end{array}\right)+\left(\begin{array}{c}
L_{0} \\
L_{1}
\end{array}\right) w \\
+\left(\begin{array}{c}
B \\
0
\end{array}\right) u+\left(\begin{array}{cc}
G & 0 \\
0 & G_{r}
\end{array}\right)\left(\begin{array}{c}
\hat{\lambda} \\
\hat{\lambda}_{r}
\end{array}\right) \\
\left(\begin{array}{c}
\hat{\lambda} \\
\hat{\lambda}_{r}
\end{array}\right) \in-\mathcal{N}_{\mathcal{S}(t) \times \mathcal{S}(t)}\left(\bar{H}\left(\begin{array}{c}
\hat{x} \\
\hat{x}_{r}
\end{array}\right)+\bar{J}\left(\begin{array}{c}
\hat{\lambda} \\
\hat{\lambda}_{r}
\end{array}\right)\right)
\end{gathered}
$$

where the gain matrix $L:=\left(\begin{array}{l}L_{0} \\ L_{1}\end{array}\right)$ will be designed in the sequel, and the matrices $\bar{H}, \bar{J}$ are defined as follows:

$$
\bar{H}:=\left(\begin{array}{cc}
H & 0 \\
0 & H_{r}
\end{array}\right), \quad \bar{J}:=\left(\begin{array}{cc}
J & 0 \\
0 & J_{r}
\end{array}\right) .
$$

For brevity, we have suppressed the time argument in (22), and will do so in the remainder of this section unless required. Let us also introduce the following notation:

$$
\bar{A}:=\left(\begin{array}{cc}
A & F \\
0 & A_{r}
\end{array}\right), \quad \bar{C}:=\left(\begin{array}{ll}
C & D
\end{array}\right), \quad \bar{G}:=\left(\begin{array}{cc}
G & 0 \\
0 & G_{r}
\end{array}\right) .
$$

Theorem 3: Consider systems (3), (4) under assumptions (A2) and (A3). Suppose that there exist a feedback matrix $K$ and an injection matrix $L$ that render the triplets $(A+B K, G, H, J)$ and $(\bar{A}-L \bar{C}, \bar{G}, \bar{H}, \bar{J})$ strictly passive, respectively. If there exist matrices $\Pi \in \mathbb{R}^{n \times d_{r}}$ and $M \in$ $\mathbb{R}^{d_{u} \times d_{r}}$ that satisfy (18), then the output regulation problem is solved by letting

$$
u(t)=K \hat{x}(t)+(M-K \Pi) \hat{x}_{r}(t) .
$$


Proof: Introduce the variable $\tilde{x}:=x-\hat{x}$, then the closed-loop dynamics are similar to

$$
\begin{aligned}
\left(\begin{array}{c}
\dot{x} \\
\dot{\tilde{x}} \\
\dot{\hat{x}}_{r}
\end{array}\right)= & \underbrace{\left(\begin{array}{ccc}
A+B K & -B K & B(M-K \Pi) \\
0 & A-L_{0} C & -F+L_{0} D \\
0 & L_{1} C & A_{r}-L_{1} D
\end{array}\right)}_{:=A_{c l}}\left(\begin{array}{c}
x \\
\tilde{x} \\
\hat{x}_{r}
\end{array}\right) \\
& +\underbrace{\left(\begin{array}{c}
F-L_{0} D \\
L_{1} D
\end{array}\right)}_{:=F_{c l}} x_{r}+\underbrace{\left(\begin{array}{ccc}
G & 0 & 0 \\
G & -G & 0 \\
0 & 0 & G_{r}
\end{array}\right)}_{:=G_{c l}}\left(\begin{array}{c}
\lambda \\
\hat{\lambda} \\
\hat{\lambda}_{r}
\end{array}\right)
\end{aligned}
$$

$$
\left(\begin{array}{c}
\lambda \\
\hat{\lambda} \\
\hat{\lambda}_{r}
\end{array}\right) \in-\mathcal{N}_{\mathcal{S}(t) \times \mathcal{S}(t) \times \mathcal{S}(t)}\left(H_{c l}\left(\begin{array}{c}
x \\
\tilde{x} \\
\hat{x}_{r}
\end{array}\right)+J_{c l}\left(\begin{array}{c}
x \\
\tilde{x} \\
\hat{x}_{r}
\end{array}\right)\right),
$$

where we used the notation $H_{c l}:=\left(\begin{array}{ccc}H & 0 & 0 \\ H & -H & 0 \\ 0 & 0 & H_{r}\end{array}\right)$, and $J_{c l}:=$ $\left(\begin{array}{lll}J & 0 & 0 \\ 0 & J & 0 \\ 0 & 0 & J_{r}\end{array}\right)$.

Well-posedness: To show that the closed-loop system admits a unique solution, we follow the same procedure as in the proof of Theorem 2. That is, we find a symmetric positive definite matrix $P_{c l}$ such that $\operatorname{ker}\left(P_{c l} G_{c l}-H_{c l}^{\top}\right) \subseteq \operatorname{ker}\left(J_{c l}+\right.$ $\left.J_{c l}^{\top}\right)$. The matrix $J_{c l}$ is positive semidefinite by construction. To find the matrix $P_{c l}$, it is noted that, by assumption, the quadruples $(A+B K, G, H, J)$ and $(\bar{A}-L \bar{C}, \bar{G}, \bar{H}, \bar{J})$ are strictly passive, so there exist symmetric positive definite matrices $P, \bar{P}$ with dissipation constants $\gamma, \bar{\gamma}$, such that (17) holds. Now, partition $\bar{P}$ as $\bar{P}:=\left(\begin{array}{ll}\bar{P}_{11} & \bar{P}_{12} \\ \bar{P}_{12} & \bar{P}_{22}\end{array}\right)$, then $\bar{P}_{22}$ is a symmetric positive definite matrix, and $\bar{P}_{22} G_{r}=H_{r}^{\top}$. Thus, by letting

$$
P_{c l}:=\left[\begin{array}{ccc}
2 P & -P & 0 \\
-P & P & 0 \\
0 & 0 & \bar{P}_{22}
\end{array}\right]
$$

it is easily checked that $P_{c l}$ is symmetric, positive definite, and $\operatorname{ker}\left(P_{c l} G_{c l}-H_{c l}^{\top}\right) \subseteq \operatorname{ker}\left(J+J_{c l}^{\top}\right)$.

Regulation: By letting $W:=(-K \quad(M-K \Pi))$, it is seen that

$$
A_{c l}=\left(\begin{array}{cc}
A+B K & B W \\
0 & \bar{A}-L \bar{C}
\end{array}\right)
$$

Define the regulation error as follows:

$$
e:=\left(\begin{array}{c}
x-\Pi x_{r} \\
\tilde{x} \\
\hat{x}_{r}-x_{r}
\end{array}\right)=:\left(\begin{array}{c}
e_{x} \\
\tilde{x} \\
e_{r}
\end{array}\right)=:\left(\begin{array}{c}
e_{x} \\
e_{\xi}
\end{array}\right),
$$

then it can be shown using the equations in (18) that

$$
\begin{aligned}
& \dot{e}=\left(\begin{array}{cc}
A+B K & B W \\
0 & \bar{A}-L \bar{C}
\end{array}\right) e \\
& +\left(\begin{array}{ccc}
G & 0 & 0 \\
0 & G & 0 \\
0 & 0 & G_{r}
\end{array}\right)\left(\begin{array}{c}
\lambda-\lambda_{r} \\
\lambda-\hat{\lambda} \\
\lambda_{r}-\hat{\lambda}_{r}
\end{array}\right)
\end{aligned}
$$

where $\lambda, \lambda_{r}$ and $\left(\hat{\lambda}, \hat{\lambda}_{r}\right)$ are defined in (3b), (4b) and (22b), respectively.
Consider the Lyapunov function $V(e)=e^{\top}\left[\begin{array}{cc}\alpha P & 0 \\ 0 & \beta \bar{P}\end{array}\right] e$ for some $\alpha, \beta>0$ to be specified later. The derivative of $V(\cdot)$ along the trajectories of the closed-loop system satisfies the following relations:

$$
\begin{aligned}
\dot{V}(e)= & \alpha e_{x}^{\top}\left(P(A+B K)+(A+B K)^{\top} P\right) e_{x} \\
& +2 \alpha e_{x}^{\top} P B W e_{\xi} \\
& +e_{\xi}^{\top}\left(\beta \bar{P}(\bar{A}-L \bar{C})+(\bar{A}-L \bar{C})^{\top} \beta \bar{P}\right) e_{\xi} \\
& +2 \alpha e_{x}^{\top} P G\left(\lambda-\lambda_{r}\right)+2 \beta e_{\xi}^{\top} \bar{P} \bar{G}\left(\begin{array}{c}
\lambda-\hat{\lambda} \\
\lambda_{r}-\hat{\lambda}_{r}
\end{array}\right) \\
\leq & -\alpha \gamma e_{x}^{\top} P e_{x}-\beta \bar{\gamma} e_{\xi}^{\top} \bar{P} e_{\xi}+2 \alpha e_{x}^{\top} P B W e_{\xi} \\
& +2 \alpha\left(\lambda-\lambda_{r}\right)^{\top}\left(H e_{x}+J\left(\lambda-\lambda_{r}\right)\right) \\
& +2 \beta(\lambda-\hat{\lambda})^{\top}(H \tilde{x}+J(\lambda-\hat{\lambda})) \\
& +2 \beta\left(\lambda_{r}-\hat{\lambda}_{r}\right)^{\top}\left(H_{r} e_{r}+J_{r}\left(\lambda_{r}-\hat{\lambda}_{r}\right)\right)
\end{aligned}
$$

where the last inequality is obtained using the passivity assumption on $(A+B K, G, H, J)$ and $(\bar{A}-L \bar{C}, \bar{G}, \bar{H}, \bar{J})$. Using the assumptions that $H \Pi=H_{r}$, and $J=J_{r}$, it now follows from the definition of the normal cone that

$$
\begin{aligned}
(\lambda & \left.-\lambda_{r}\right)^{\top}\left(H e_{x}+J\left(\lambda-\lambda_{r}\right)\right) \\
& =\left\langle\lambda-\lambda_{r}, H x-H \Pi x_{r}+J \lambda-J_{r} \lambda_{r}\right\rangle \\
& =\left\langle\lambda-\lambda_{r}, H x+J \lambda-H_{r} x_{r}-J_{r} \lambda_{r}\right\rangle \leq 0
\end{aligned}
$$

and using similar arguments,

$$
\begin{aligned}
& (\lambda-\hat{\lambda})^{\top}(H \tilde{x}+J(\lambda-\hat{\lambda})) \leq 0 \\
& \left(\lambda_{r}-\hat{\lambda}_{r}\right)^{\top}\left(H_{r} e_{r}+J_{r}\left(\lambda_{r}-\hat{\lambda}_{r}\right)\right) \leq 0 .
\end{aligned}
$$

Plugging these relations in the expression for $\dot{V}(\cdot)$, and using the notation $\sigma_{\min }$ to denote the smallest eigenvalue of a matrix and $\chi$ to denote the induced Euclidean matrix norm of $P B W$, we get

$$
\begin{aligned}
\dot{V}(e) \leq- & \alpha \gamma \sigma_{\min }(P)\left|e_{x}\right|^{2}-\beta \bar{\gamma} \sigma_{\min }(\bar{P})\left|e_{\xi}\right|^{2} \\
& +2 \alpha \chi\left|e_{x}\right|\left|e_{\xi}\right| \\
\leq- & \alpha \gamma \sigma_{\min }(P)\left|e_{x}\right|^{2}-\beta \bar{\gamma} \sigma_{\min }(\bar{P})\left|e_{\xi}\right|^{2}+\left|e_{x}\right|^{2} \\
& +\alpha^{2} \chi^{2}\left|e_{\xi}\right|^{2} \\
= & \left(\alpha \gamma \sigma_{\min }(P)-1\right)\left|e_{x}\right|^{2} \\
& -\left(\beta \bar{\gamma} \sigma_{\min }(\bar{P})-\alpha^{2} \chi^{2}\right)\left|e_{\xi}\right|^{2} .
\end{aligned}
$$

Thus, choosing $\alpha, \beta$ in the definition of the Lyapunov function $V(\cdot)$ such that $\alpha \gamma \sigma_{\min }(P)>1$ and $\beta \bar{\gamma} \sigma_{\min }(\bar{P})>$ $\alpha^{2} \chi^{2}$ ), makes $\dot{V}(\cdot)$ negative definite. In particular $e_{x}$ converges to zero, from which it follows that $w=C x+D x_{r}=$ $C\left(x-\Pi x_{r}\right)=C e_{x}$ converges to zero.

Closed-loop stabilization: The closed-loop stabilization could be shown in exactly the same manner as above. The difference being, the derivative of the Lyapunov function $V(\cdot)$ is calculated along the trajectories of the dynamics of $\left(x, \tilde{x}, \hat{x}_{r}\right)$ given in (24).

\section{CONClusions}

This paper studied the problem of output regulation in a certain class of nonsmooth dynamical systems that are modeled as differential variational inequalities. These systems 
in particular model systems where states are constrained to evolve within some closed, convex and time-varying set. We first studied the conditions under which there exists a unique solution for such systems. The classical internal model principle was then used to derive conditions to synthesize a control law that achieves the desired objective. The analysis were based on using the Lyapunov methods in combination with monotonicity property of the normal cone operator to prove stability.

Several extensions are possible. The most interesting of which is to consider cases where the set-valued mapping $\mathcal{S}(\cdot)$ is not just time-dependent, but also state-dependent. This would allow us to model mechanical systems with impacts within our framework but deeper investigation is required for the synthesis of control in such cases.

\section{APPENDIX}

Proof of Lemma 1: First, it is noted that $\sigma_{\mathcal{S}(t)}$ is sublinear [22, Theorem 8.24], and hence a convex functional, so that $\partial \sigma_{\mathcal{S}(t)}$ is maximal monotone for each $t \geq 0$, and $\operatorname{rint}\left(\operatorname{dom} \partial \sigma_{\mathcal{S}(t)}\right) \neq \emptyset$ because $\mathcal{S}(t)$ is convex-valued and $\operatorname{rint}(\mathcal{S}(t)) \neq \emptyset$. The matrix $J$ define a monotone mapping, and being continuous, is also maximal monotone [22, Example 12.7]. Also, $\operatorname{dom} J$ is $\mathbb{R}^{d_{s}}$. It now holds that $\left(\partial \sigma_{\mathcal{S}(t)}+J\right)$ is maximal monotone because $\operatorname{rint}\left(\operatorname{dom} \partial \sigma_{\mathcal{S}(t)}\right) \cap \operatorname{rint}(\operatorname{dom} J) \neq \emptyset$ [22, Corollary 12.44]. Hence, $\left(\partial \sigma_{\mathcal{S}(t)}+J\right)^{-1}$ is also maximal monotone, because the inverse operation preserves maximal monotonicity [22, Exercise 12.8(a)]. Also, using the fact that $\operatorname{rge}(\mathcal{S}(t))+J)=$ $\operatorname{rge}(\mathcal{S}(t))+\operatorname{rge}(J)$, and that $\operatorname{dom} \Phi(t, \cdot)=\operatorname{rge}\left(\partial \sigma_{\mathcal{S}(t)}+J\right)$, the desired result follows.

Proof of Lemma 2: For $i=1,2$, consider $\eta_{i} \in$ $\left(\partial \sigma_{\mathcal{S}(t)}+J\right)^{-1}(H x)$, then $H x-J \eta_{i} \in \partial \sigma_{\mathcal{S}(t)}\left(\eta_{i}\right)$. Since $\partial \sigma_{\mathcal{S}(t)}$ is monotone, we have

$$
\left\langle H x-J \eta_{1}-H x+J \eta_{2}, \eta_{1}-\eta_{2}\right\rangle \geq 0
$$

or

$$
\left\langle J\left(\eta_{1}-\eta_{2}\right), \eta_{1}-\eta_{2}\right\rangle \leq 0
$$

It follows that $\eta_{1}-\eta_{2} \in \operatorname{ker}\left(J+J^{\top}\right)$, because $J$ is positive semidefinite.

Proof of Lemma 3: From Lemma 2, it directly follows that the operator $\mathcal{P}_{J}(\Phi(t, H \cdot))$ is single-valued. Let $\eta^{j}:=$ $\mathcal{P}_{J}\left(\Phi\left(t, H x^{j}\right)\right)$, for $j=a, b$, so that $H x^{j}-J z^{j} \in \partial \sigma_{\mathcal{S}}\left(\eta^{j}\right)$. Monotonicity of $\partial \sigma_{\mathcal{S}}$ gives

$$
\left\langle H x^{a}-J \eta^{a}-H x^{b}+J \eta^{b}, \eta^{a}-\eta^{b}\right\rangle \geq 0
$$

or equivalently,

$$
\left\langle J\left(\eta^{a}-\eta^{b}\right), \eta^{a}-\eta^{b}\right\rangle \leq\left\langle H\left(x^{a}-x^{b}\right), \eta^{a}-\eta^{b}\right\rangle .
$$

Since $J$ is positive semidefinite, there exists $c>0$, such that

$$
\left|\mathcal{P}_{J}\left(\eta^{a}\right)-\mathcal{P}_{J}\left(\eta^{b}\right)\right| \leq c\left\langle J\left(\eta^{a}-\eta^{b}\right), \eta^{a}-\eta^{b}\right\rangle .
$$

Assume now that, $\mathcal{P}_{J}(\Phi(t, H \cdot))$ is not Lipschitz, so for every $i \in \mathbb{N}$, there exist pairs $\left(x_{i}^{a}, x_{i}^{b}\right), x_{i}^{a} \neq x_{i}^{b}$, and $\eta_{i}^{a} \in \Phi\left(t, H x_{i}^{a}\right)$, and $\eta_{i}^{b} \in \Phi\left(t, H x_{i}^{b}\right)$, such that

$$
\begin{aligned}
i\left|x_{i}^{a}-x_{i}^{b}\right| & <\left|\mathcal{P}_{J}\left(\eta_{i}\right)-\mathcal{P}_{J}\left(\eta_{i}^{b}\right)\right| \\
& \leq c\left\langle J\left(\eta_{i}^{a}-\eta_{i}^{b}\right), \eta_{i}^{a}-\eta_{i}^{b}\right\rangle \\
& \leq c\left\langle H\left(x_{i}^{a}-x_{i}^{b}\right), \eta_{i}^{a}-\eta_{i}^{b}\right\rangle \\
& \leq c\left|x_{i}^{a}-x_{i}^{b}\right| \cdot\left|H^{\top}\left(\eta_{i}^{a}-\eta_{i}^{b}\right)\right| .
\end{aligned}
$$

Thus, we must have $\left|\eta_{i}^{a}-\eta_{i}^{b}\right| \geq\left|H^{\top}\left(\eta_{i}^{a}-\eta_{i}^{b}\right)\right| i$. Now dividing (27) by $\left|\eta_{i}^{a}-\eta_{i}^{b}\right|^{2}$, we get

$$
\left\langle J \frac{\left(\eta_{i}^{a}-\eta_{i}^{b}\right)}{\left|\eta_{i}^{a}-\eta_{i}^{b}\right|}, \frac{\eta_{i}^{a}-\eta_{i}^{b}}{\left|\eta_{i}^{a}-\eta_{i}^{b}\right|}\right\rangle \leq\left\langle\frac{x_{i}^{a}-x_{i}^{b}}{\left|\eta_{i}^{a}-\eta_{i}^{b}\right|}, \frac{H^{\top}\left(\eta_{i}^{a}-\eta_{i}^{b}\right)}{\left|\eta_{i}^{a}-\eta_{i}^{b}\right|}\right\rangle .
$$

Since the right-hand side converges to zero as $i$ gets large, it follows that $\left\langle J\left(\eta_{i}^{a}-\eta_{i}^{b}\right), \eta_{i}^{a}-\eta_{i}^{b}\right\rangle$ converges to zero as well because $J$ is positive semidefinite. But then $\mid \mathcal{P}_{J}\left(\eta_{i}\right)-$ $\mathcal{P}_{J}\left(\eta_{i}^{b}\right) \mid$ converges to zero, which is a contradiction, and the desired claim follows.

Proof of Lemma 5: If $\bar{\mu}=0$ in (16), then the result follows directly from [18, Theorem 3]. Next, for $i=1,2$, consider the differential inclusion

$$
\dot{z}_{i}(t) \in-\bar{\Psi}\left(t, z_{i}\right)+g_{i}(t)
$$

for some locally absolutely continuous functions $g_{i}(\cdot)$. It again follows from [18, Theorem 3] that there exists a unique solution $z_{i}(\cdot)$, which is locally absolutely continuous. It holds due to monotonicity of $\bar{\Psi}(t, \cdot)$ that

$$
\begin{aligned}
\frac{1}{2} \frac{d}{d t}\left|z_{1}(t)-z_{2}(t)\right|^{2} & =\left\langle\dot{z}_{1}(t)-\dot{z}_{2}(t), z_{1}(t)-z_{2}(t)\right\rangle \\
& \leq\left\langle g_{1}(t)-g_{2}(t), z_{1}(t)-z_{2}(t)\right\rangle \\
& \leq\left|g_{1}(t)-g_{2}(t)\right| \cdot\left|z_{1}(t)-z_{2}(t)\right|
\end{aligned}
$$

Letting $Z(t):=\left|z_{1}(t)-z_{2}(t)\right|^{2}$, the above inequality is rewritten as: $\dot{Z}(t) \leq 2\left|g_{1}(t)-g_{2}(t)\right| \sqrt{Z(t)}$. Applying the comparison lemma for solution of ODEs, we get

$$
\left|z_{1}(t)-z_{2}(t)\right| \leq\left|z_{1}(0)-z_{2}(0)\right|+\int_{0}^{t}\left|g_{1}(s)-g_{2}(s)\right| d s .
$$

To study the solutions of (16), consider a sequence of solutions with $z_{1}(t)=z(0), t \geq 0$, and $z_{i+1}(\cdot)$, for $i \geq 1$ is obtained as a solution to following inclusion with initial condition $z_{i}(0)=z(0), i \in \mathbb{N}$ :

$$
\dot{z}_{i+1}(t) \in-\bar{\Psi}\left(t, z_{i+1}\right)+\bar{\mu} z_{i}(t) .
$$

Then, from (28), we have, for each $t \geq 0$ :

$$
\left|z_{i+1}(t)-z_{i}(t)\right| \leq \int_{0}^{t} \bar{\mu}\left|z_{i}(s)-z_{i-1}(s)\right| d s
$$

which through induction leads to:

$$
\left|z_{i+1}(t)-z_{i}(t)\right| \leq \frac{(\bar{\mu} t)^{i}}{i !}\left\|z_{2}-z_{1}\right\|_{\mathcal{L}_{\infty}}
$$

Thus, the sequence $\left\{z_{i}\right\}_{i=1}^{\infty}$ converges uniformly on every compact interval, and hence $\lim _{i \rightarrow \infty} z_{i}$ is the unique solution to system (16). 


\section{REFERENCES}

[1] V. Acary, O. Bonnefon and B. Brogliato. Nonsmooth Modeling and Simulation for Switched Circuits, volume 69 of Lecture Notes in Electrical Engineering, Springer, Heidelberg, 2011.

[2] J.-P. Aubin and A. Cellina. Differential Inclusions. Springer, New York, 1984.

[3] H. Brézis. Opérateurs Maximaux Monotones et Semi-Groupes des Contractions dans les Espaces de Hilbert. North-Holland, Mathematics Studies, 1973.

[4] B. Brogliato. Some results on optimal control with unilateral state constraints. Nonlinear Analysis: Theory, Methods, and Applications, 70(10):3626 - 3657, 2009.

[5] B. Brogliato and D. Goeleven. Well-posedness, stability and invariance results for a class of multivalued Lur'e dynamical systems. Nonlinear Analysis Ser. A: Theory, Methods \& Applications, 74:195 - 212, 2011.

[6] B. Brogliato and W.P.M.H. Heemels. Observer design for Lur'e systems with multivalued mappings: A passivity approach. IEEE Trans. Automatic Control, 54(8):1996 - 2001, 2009.

[7] B. Brogliato and L. Thibault. Existence and uniqueness of solutions for non-autonomous complementarity dynamical systems. Journal of Convex Analysis, 17(3 \& 4):961 - 990, 2010.

[8] M.K. Camlibel, W.P.M.H. Heemels, and J.M. Schumacher. On linear passive complementarity systems. European J. of Control, 8(3):220 237, 2002.

[9] M.K. Camlibel, J.-S. Pang, and J. Shen. Lyapunov stability of complementarity and extended systems. SIAM J. Optim., 17(4):1056 - 1101, 2006.

[10] M.K. Camlibel and J.M. Schumacher. Linear passive systems and maximal monotone mappings. Submitted for publication.

[11] D. Carnevale, S. Galeani, L. Menini and M. Sassano. Robust semiclassical internal model based regulation for a class of hybrid linear systems. In Proc. 19th IFAC World Congress, South Africa, 2014, pp. 1519 - 1524.

[12] N. Cox, L. Marconi, and A. Teel. High-gain observers and linear output regulation for hybrid exosystems. Int. J. Robust \& Nonlinear Control, 24(6): 1043 - 1063, 2014.

[13] F. Facchinei and J.-S. Pang. Finite-Dimensional Variational Inequalities and Complementarity Problems. Springer, 2003.

[14] B. Francis. A linear multivariable regulator problem. SIAM J. Control and Optimization, 15(3):486 - 505, 1977.

[15] S. Galeani, L. Menini, and A. Potini. Robust trajectory tracking for a class of hybrid systems: An internal model principle approach. IEEE Trans. Automatic Control, 57(2): 344 - 359, 2012.

[16] D. Goeleven and B. Brogliato. Stability and instability matrices for linear evolution variational inequalities. IEEE Trans. Automatic Control, 49(4):521 - 534, 2004.

[17] W.P.M.H. Heemels, M.K. Camlibel, J.M. Schumacher, and B. Brogliato. Observer-based control of linear complementarity systems. Int. J. Robust \& Nonlin. Control, 21(10):1193-1218, 2011.

[18] M. Kunze and M.D.P. Monteiro Marques. BV solutions to evolution problems with time-dependent domains. Set-Valued Analysis, 5:57 72, 1997.

[19] L. Marconi and A. Teel. Internal model principle for linear systems with periodic state jumps. IEEE Trans. Automatic Control, 58(11): 2788 - 2802, 2013.

[20] M.D.P. Monteiro Marques. Differential Inclusions in Nonsmooth Mechanical Problems: Shocks and Dry Friction, vol. 9 of Progress in Nonlinear Differential Equations and their Applications. Birkhäuser, 1993.

[21] J.-S. Pang and D.E. Stewart. Differential variational inequalities. Math. Prog., Ser. A, 113:345 - 424, 2008.

[22] R.T. Rockafellar and R.J.-B. Wets. Variational Analysis, volume 317 of Gundlehren der mathematischen Wissenchaften. Springer-Verlag, Berlin, 3rd printing, 2009 edition, 1998.

[23] A. Tanwani, B. Brogliato, and C. Prieur. Passivity-based observer design for a class of Lagrangian systems with perfect unilateral constraints. In Proc. 52nd IEEE Conf. Decision \& Control, Italy, 2013, pp. $3338-3343$.

[24] A. Tanwani, B. Brogliato, and C. Prieur. Stability and observer design for multivalued Lur'e systems with non-monotone, time-varying nonlinearities and state jumps. To appear in SIAM J. Control and Optimization, 2014.

[25] A. Tanwani, B. Brogliato, and C. Prieur. On output regulation in stateconstrained systems: An application to polyhedral case. In Proc. 19th IFAC World Congress, South Africa, 2014, pp. 1513 - 1518.
[26] F. Vasca, M.K. Camlibel, L. Iannelli, and R. Frasca. A new perspective for modeling power electronics converters: Complementarity framework. IEEE Trans. on Power Electronics, 24(2):456 - 468, 2009. 